\title{
A theoretical investigation of the feasibility of Tannor-Rice type control: Application to selective bond breakage in gas-phase dihalomethanes
}

Shu, Chuan-Cun; Rozgonyi, Tamas; Gonzalez, Leticia; Henriksen, Niels Engholm

Published in:

Journal of Chemical Physics

Link to article, DOI:

$10.1063 / 1.4706603$

Publication date:

2012

Document Version

Publisher's PDF, also known as Version of record

Link back to DTU Orbit

Citation (APA):

Shu, C-C., Rozgonyi, T., Gonzalez, L., \& Henriksen, N. E. (2012). A theoretical investigation of the feasibility of Tannor-Rice type control: Application to selective bond breakage in gas-phase dihalomethanes. Journal of Chemical Physics, 136(17), 174303. https://doi.org/10.1063/1.4706603

\section{General rights}

Copyright and moral rights for the publications made accessible in the public portal are retained by the authors and/or other copyright owners and it is a condition of accessing publications that users recognise and abide by the legal requirements associated with these rights.

- Users may download and print one copy of any publication from the public portal for the purpose of private study or research.

- You may not further distribute the material or use it for any profit-making activity or commercial gain

- You may freely distribute the URL identifying the publication in the public portal 


\section{AIP $\begin{gathered}\text { mosoumator } \\ \text { chemical Physics }\end{gathered}$}

A theoretical investigation of the feasibility of Tannor-Rice type control: Application to selective bond breakage in gas-phase dihalomethanes

Chuan-Cun Shu, Tamás Rozgonyi, Leticia González, and Niels E. Henriksen

Citation: J. Chem. Phys. 136, 174303 (2012); doi: 10.1063/1.4706603

View online: http://dx.doi.org/10.1063/1.4706603

View Table of Contents: http://jcp.aip.org/resource/1/JCPSA6/v136/i17

Published by the American Institute of Physics.

Additional information on J. Chem. Phys.

Journal Homepage: http://jcp.aip.org/

Journal Information: http://jcp.aip.org/about/about_the_journal

Top downloads: http://jcp.aip.org/features/most_downloaded

Information for Authors: http://jcp.aip.org/authors

\section{ADVERTISEMENT}

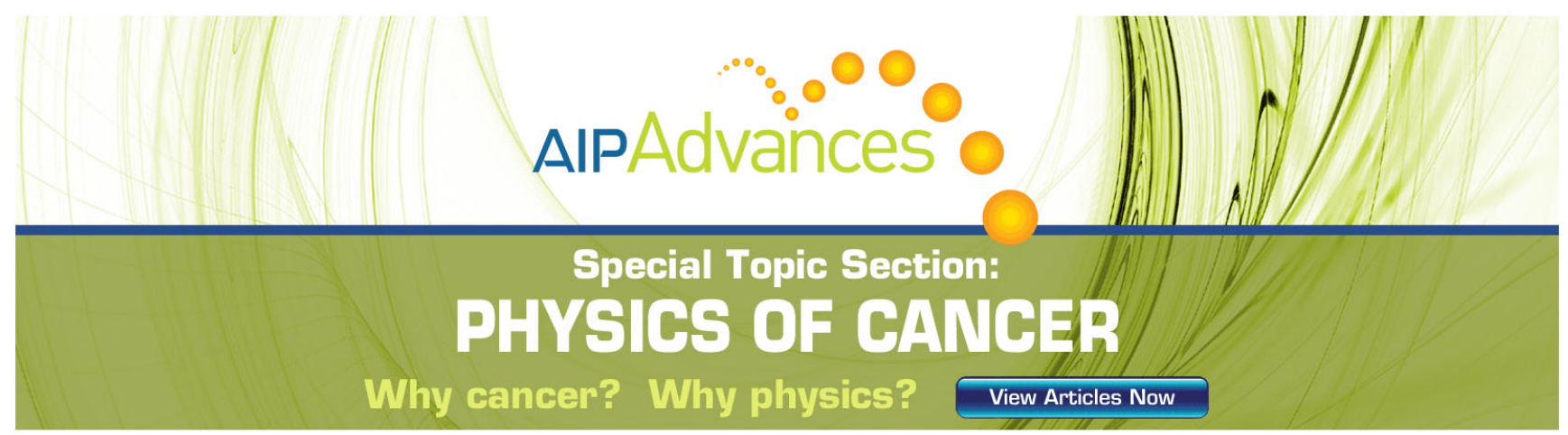




\title{
A theoretical investigation of the feasibility of Tannor-Rice type control: Application to selective bond breakage in gas-phase dihalomethanes
}

\author{
Chuan-Cun Shu, ${ }^{1}$ Tamás Rozgonyi, ${ }^{2}$ Leticia González, ${ }^{3}$ and Niels E. Henriksen ${ }^{1, a)}$ \\ ${ }^{1}$ Department of Chemistry, Building 207, Technical University of Denmark, DK-2800 Kgs. Lyngby, Denmark \\ ${ }^{2}$ Research Centre for Natural Sciences, Hungarian Academy of Sciences, 1025 Budapest, \\ Pusztaszeri út 59-67, Hungary \\ ${ }^{3}$ Institute of Theoretical Chemistry, University of Vienna, Währinger Str. 17, 1090 Vienna, Austria
}

(Received 12 January 2012; accepted 11 April 2012; published online 1 May 2012)

\begin{abstract}
Within the $\tilde{\mathrm{B}}$ absorption band of $\mathrm{CH}_{2} \mathrm{BrCl}$, we theoretically analyze the laser-induced control of the $\mathrm{Br} / \mathrm{Cl}$ branching ratio, $\mathrm{Br}+\mathrm{CH}_{2} \mathrm{Cl} \leftarrow \mathrm{CH}_{2} \mathrm{BrCl} \rightarrow \mathrm{CH}_{2} \mathrm{Br}+\mathrm{Cl}$, with $\mathrm{CH}_{2} \mathrm{BrCl}$ initially in its vibrational ground state. For weak-field excitation, the $\mathrm{Br} / \mathrm{Cl}$ branching ratio increases as a function of wavelength, however, for wavelengths below $180 \mathrm{~nm}$ the branching ratio cannot be made smaller than 0.4. Using optimal control theory, we show that the branching ratio can be made significantly less than 0.4 , only when very strong fields are employed. Thus, the present work strongly suggests that a Tannor-Rice type laser control mechanism for selective bond breakage in $\mathrm{CH}_{2} \mathrm{BrCl}$ cannot take place without accompanying photoionization. (ㅇ 2012 American Institute of Physics. [http://dx.doi.org/10.1063/1.4706603]
\end{abstract}

\section{INTRODUCTION}

Beyond simple wavelength tuning, several approaches for quantum control with laser pulses have been developed. ${ }^{1-6}$ One such approach is the Tannor-Rice pump-dump scheme for controlling the selectivity of product formation in a chemical reaction. ${ }^{1-3}$ In the original version of this scheme an excited electronic state was used to mediate reaction on a ground electronic state potential energy surface (PES). This was done via pump and dump of nuclear wave packets between the electronic states using ultrashort laser pulses with a fixed pulse shape and a variable time delay. In this work we focus on the feasibility of a similar Tannor-Rice type of control where ultrashort laser pulses (with optimized pulse shapes) create and guide wave packets via properly timed pump and dump pulses between various potential energy surfaces. The key point in this scheme is that wave packet motion can be guided by taking advantage of the different forces which operate in various electronic states.

Theoretically, the most general framework used to address quantum control problems is optimal control theory (OCT) that enables attaining the quantum dynamical objective in the best possible way. ${ }^{7-9}$ This approach was already applied to the original version of the TannorRice pump-dump scheme. ${ }^{3}$ Much effort, including the wellknown Krotov method, ${ }^{10,11}$ the Zhu-Rabitz method, ${ }^{12,13}$ and a recently formulated two-point boundary-value quantum control paradigm (TBQCP), ${ }^{14,15}$ has gone into developing efficient schemes to deal with the iterative process of searching for optimized control fields. In practice, the resultant optimal control fields often require further frequency filtering, which could in turn hinder the convergence rate or even the monotonicity. ${ }^{8}$ Recently, a "fast-kick-off" algorithm ${ }^{16}$ for

\footnotetext{
a)Electronic mail: neh@kemi.dtu.dk.
}

searching optimal fields has been developed on the basis of the TBQCP method. The new algorithm is expected to effectively improve the search efficiency and to attain good monotonic convergence quality, especially, when frequency constraints are considered.

There are a number of applications of OCT ranging from the control of chemical reactions ${ }^{3,17}$ to quantum optical problems, such as quantum information processing ${ }^{18-20}$ or the preparation of cold molecules. ${ }^{21,22}$ However, the number of theoretical studies where high quality potential energy surfaces including realistic transition dipole moment surfaces are employed is quite limited. To that end, we studied recently selective bond breakage in HOD, $\mathrm{D}+\mathrm{OH} \leftarrow \mathrm{HOD} \rightarrow \mathrm{H}+\mathrm{OD}$, within the first absorption band via optimized ultraviolet laser pulses. ${ }^{17}$ The molecule was initially assumed to be in its vibrational ground state, which is the typical situation in current experiments with shaped laser pulses. ${ }^{23}$ We obtained in the strong-field limit (peak intensities $\sim 10 \mathrm{TW} / \mathrm{cm}^{2}$ ) a $\mathrm{OD} / \mathrm{OH}$ branching ratio significantly less than the value of 2 , which is the smallest branching ratio which can be obtained in the weak-field limit, using any form of UV-pulse shaping control. The optimized pulses lead to a pump-dump-pump mechanism. That is, preferred cleavage of the O-D bond is obtained via the creation of a nuclear wave packet on the isolated potential energy surface of the first absorption band of water, followed by wave packet dumping to the electronic ground state, creating a nonstationary vibrational state in HOD and, finally, this wave packet is pumped into the first absorption band.

In the present work we will study the chemically more interesting problem of selective bond breakage in $\mathrm{CH}_{2} \mathrm{BrCl}$ within the second absorption band. The dynamics-which includes nonadiabatic transitions between the first and second excited electronic states-is considerably more complicated than in HOD. Dihalomethanes, such as $\mathrm{CH}_{2} \mathrm{BrCl}$ and 
$\mathrm{CH}_{2} \mathrm{BrI}$, are interesting candidates to explore the complex interplay of competing dynamical fragmentation pathways, from both the theoretical and experimental point of view. ${ }^{24-26}$ An early approach to achieve selective bond breakage involved wavelength tuning of narrow-band UV laser sources to excite electronic transitions to the dissociation continua of selected bonds. Successful application has been demonstrated for the gas-phase photoproduct distribution of $\mathrm{CH}_{2} \mathrm{BrI}$, where excitation at $210 \mathrm{~nm}$ resulted in exclusive fission of the $\mathrm{C}-\mathrm{Br}$ bond, while excitation at $248 \mathrm{~nm}$ resulted in preferred cleavage of the $\mathrm{C}-\mathrm{I}$ bond. ${ }^{27}$ Quantum mechanical computations using empirical potential energy surfaces could essentially reproduce these findings. ${ }^{28}$ Furthermore, it was shown that at $248 \mathrm{~nm}$ the branching ratio could be controlled beyond the experimental result reported above, by applying so-called bichromatic coherent control starting from a superposition of vibrational states of $\mathrm{CH}_{2} \mathrm{BrI}^{28}$

As mentioned above, in this work we focus on $\mathrm{CH}_{2} \mathrm{BrCl}$. Absorption of a UV photon gives rise to the production of halogen atoms and halomethyl radicals. ${ }^{26,29,30}$ Recently, accurate potential energy surfaces of $\mathrm{CH}_{2} \mathrm{BrCl}$ for the ground and the lowest two excited states including the nonadiabatic couplings and transition dipole moments have been calculated. ${ }^{31}$ This has laid the foundation for detailed quantum dynamical studies of the UV photodissociation dynamics of $\mathrm{CH}_{2} \mathrm{BrCl}$. Thus, the dependence of the $\mathrm{Br} / \mathrm{Cl}$ branching ratio

$$
\mathrm{CH}_{2} \mathrm{BrCl} \rightarrow\left\{\begin{array}{l}
\mathrm{Br}+\mathrm{CH}_{2} \mathrm{Cl} \\
\mathrm{CH}_{2} \mathrm{Br}+\mathrm{Cl}
\end{array}\right.
$$

on the wavelength of light has been examined in a recent study. ${ }^{32}$ There it was shown that, within the $\tilde{A}$ absorption band associated with the first electronically excited state, essentially complete dissociation into $\mathrm{Br}+\mathrm{CH}_{2} \mathrm{Cl}$ is observed in less than $100 \mathrm{fs}$. In the $\tilde{\mathrm{B}}$ band the dynamics takes place within the second and the first electronically excited state, mediated by nonadiabatic population transfer in about $30 \mathrm{fs}$. Since no dissociation is observed in the second adiabatic excited state, $\mathrm{CH}_{2} \mathrm{Br}+\mathrm{Cl}$ dissociation only occurs if the molecule is transferred down to the 1 st excited state from the 2 nd excited state. Accordingly, theoretical simulations using wavelengths around $200 \mathrm{~nm}$ predict 20 times more $\mathrm{Br}$ than $\mathrm{Cl}$, in good agreement with the experimental fact that no $\mathrm{CH}_{2} \mathrm{Br}+\mathrm{Cl}$ is detected. For wavelengths below $180 \mathrm{~nm}$, however, the $\mathrm{Br} / \mathrm{Cl}$ branching ratio cannot be made smaller than 0.4 by simply tuning the wavelength. Decreasing this ratio is thus a challenge for quantum control.

The question we address in this paper is whether pump and dump of wave packets between the $\tilde{B}$ band and the electronic ground state-using shaped fields as obtained by OCT - could lead to breakage of the $\mathrm{C}-\mathrm{Cl}$ bond of $\mathrm{CH}_{2} \mathrm{BrCl}$, with a selectivity beyond what is obtainable with narrowband UV lasers in the weak-field limit. Successful control in this context implies cooperative interaction between a timedependent electric field of the laser pulse and the dynamics of the molecule to alter the product distribution in the desired way.

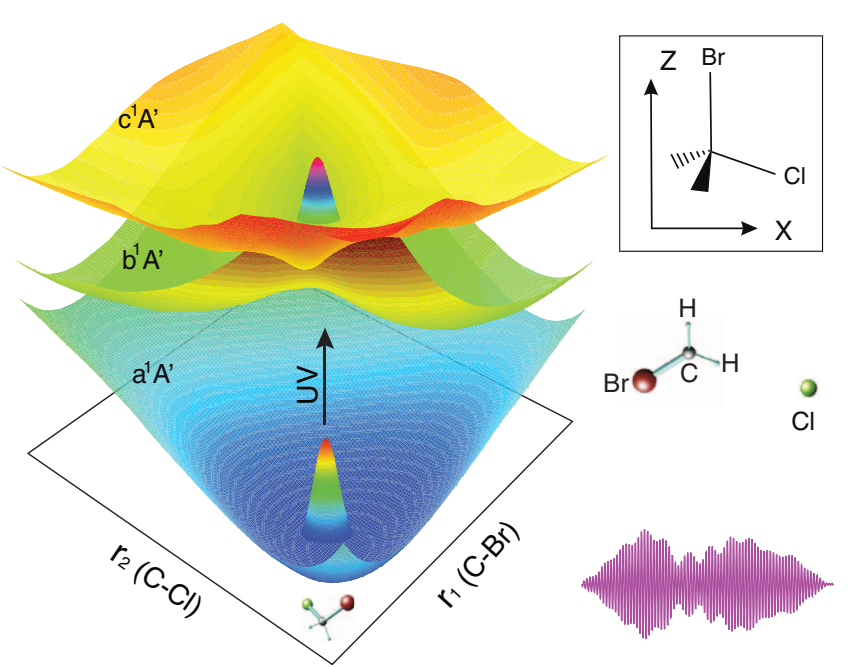

FIG. 1. Schematic diagram for optimal control of photodissociation of $\mathrm{CH}_{2} \mathrm{BrCl}$ and the adiabatic potential surfaces. The molecule is assumed to be pre-oriented in the laboratory frame, as illustrated in the inset.

The paper is organized as follows: In Sec. II we present the theoretical description of the molecule and the optimal control approach which is used in order to find the desired shaped laser pulses. In Sec. III the results are presented and discussed, and the paper is summarized in Sec. IV.

\section{THEORETICAL APPROACH}

\section{A. Quantum dynamical description}

To simplify the calculations, we treat $\mathrm{CH}_{2} \mathrm{BrCl}$ as a pseudo-triatomic molecule, composed of $\mathrm{Cl}, \mathrm{CH}_{2}$, and $\mathrm{Br}$, assuming that the higher frequency $\mathrm{C}-\mathrm{H}$ motions are frozen. We consider a two degrees of freedom model of $\mathrm{CH}_{2} \mathrm{BrCl}$, with the two bond lengths $r_{1}=\mathrm{d}(\mathrm{C}-\mathrm{Br})$ and $r_{2}=\mathrm{d}(\mathrm{C}-\mathrm{Cl})$, and we restrict ourselves to a three-state system which consists of the electronic ground state and the two lowest singlet $A^{\prime}$ states contributing to the $\tilde{\mathrm{A}}$ and $\tilde{\mathrm{B}}$ absorption bands of $\mathrm{CH}_{2} \mathrm{BrCl}$. In the adiabatic representation, we call these states $\mathrm{a}^{1} A^{\prime}, \mathrm{b}^{1} A^{\prime}$, and $\mathrm{c}^{1} A^{\prime}$, as shown in Fig. 1. The Hamiltonian can be described in terms of the two bond lenghts $r_{1}, r_{2}$ and the fixed bond angle $\theta=113.5^{\circ}$ between the carbon-halogen bonds (sometimes denoted as Eckart bond coordinates ${ }^{33}$ ).

It is convenient to describe the photodissociation dynamics within a diabatic representation of the electronic states. In the presence of an external field $\mathcal{E}(t)$, the diabatic Hamiltonian can be written as

$$
\begin{aligned}
\hat{H}_{i j}\left(r_{1}, r_{2}, \mathcal{E}(t)\right)= & -\frac{\hbar^{2} \partial^{2}}{2 m_{1} \partial^{2} r_{1}}-\frac{\hbar^{2} \partial^{2}}{2 m_{2} \partial^{2} r_{2}}-\frac{\cos \theta}{m_{3}} \frac{\hbar^{2} \partial^{2}}{\partial r_{1} \partial r_{2}} \\
& +V_{i j}\left(r_{1}, r_{2}\right)-\mu_{i j}\left(r_{1}, r_{2}\right) \mathcal{E}(t)
\end{aligned}
$$

with the reduced masses

$$
\begin{aligned}
& m_{1}=M_{\mathrm{Br}} M_{\mathrm{CH}_{2}} /\left(M_{\mathrm{Br}}+M_{\mathrm{CH}_{2}}\right), \\
& m_{2}=M_{\mathrm{Cl}} M_{\mathrm{CH}_{2}} /\left(M_{\mathrm{Cl}}+M_{\mathrm{CH}_{2}}\right), \\
& m_{3}=M_{\mathrm{CH}_{2}},
\end{aligned}
$$


where $M_{\mathrm{Br}}, M_{\mathrm{Cl}}$, and $M_{\mathrm{CH}_{2}}$ are the masses of atoms $\mathrm{Br}, \mathrm{Cl}$ (we use in the following the average atomic masses of these atoms), and that of the radical $\mathrm{CH}_{2}$, respectively. For $i=j$, $V_{i j}\left(r_{1}, r_{2}\right)$ are the diabatic PESs. The off-diagonal elements $V_{i j}\left(r_{1}, r_{2}\right)(i \neq j)$ are the potential couplings between the crossing electronic states, and $\mu_{i j}\left(r_{1}, r_{2}\right)$ are the transition dipole moments between the diabatic states. The diabatic potentials $V_{i i}\left(r_{1}, r_{2}\right)$ obtained from ab initio adiabatic potentials using approximated kinetic couplings $\left(\Gamma=40 \AA^{-1}\right.$ in Ref. 32) are labeled by $V_{i}\left(r_{1}, r_{2}\right)$ with the index $i=0,1$, and 2 in the following. We invoke these diabatic potentials, couplings, and transition dipole moments from Ref. 32 to perform wave packet propagations. We assume that the molecules are pre-oriented (see, e.g., Ref. 34) such that the $\mathrm{Cl}-\mathrm{C}-\mathrm{Br}$ atoms lie in the $\mathrm{XZ}$ plane of the laboratory system and the $\mathrm{C}-\mathrm{Br}$ bond is parallel to the Z-axis (see Fig. 1). Thus, the dipole moments $\mu_{i j}\left(r_{1}, r_{2}\right)$ have two non-zero components, $\mu_{i j}^{x}\left(r_{1}, r_{2}\right)$ and $\mu_{i j}^{z}\left(r_{1}, r_{2}\right)$. Furthermore, we assume that the electric field of the laser pulse is polarized in the $\mathrm{Z}$ direction, parallel to the $\mathrm{C}-\mathrm{Br}$ vector. Thus, the transition dipole moments $\mu_{01}^{z}\left(r_{1}, r_{2}\right)$ and $\mu_{02}^{z}\left(r_{1}, r_{2}\right)$ are included in our calculations as done in previous works ${ }^{32}$ while the rest of the elements of the dipole moments $\mu_{i j}^{z}\left(r_{1}, r_{2}\right)$ are set to zero. It should be noted that $a b$ initio values for the transition dipole moments are restricted to the Franck-Condon (FC) region, that is, $1.86 \AA \leq$ $r_{1} \leq 2.05 \AA$ and $1.68 \AA \leq r_{2} \leq 1.88 \AA$. At every point outside this region the corresponding ab initio value at the closest point on the boundary of the FC region was used. ${ }^{32}$

\section{B. Optimal control theory}

OCT is formulated as the problem of maximizing a functional that quantifies to what extent the given objective is met by a certain laser field. For the present study, we employ a scheme based on the fast-kick-off TBQCP method ${ }^{16}$ to find the laser pulse $\mathcal{E}(t)$, which can steer the quantum system of $\mathrm{CH}_{2} \mathrm{BrCl}$ from its initial state $\Phi_{i}$ to a "target" state $\Phi_{f}$ at final time $T$, so as to attain the objective of maximizing the expectation value of a target operator $\mathrm{O}$ in the $\mathrm{CH}_{2} \mathrm{Br}+\mathrm{Cl}$ channel.

The wave function $\Psi(t)$ is propagated from $t$ to $T$ under the influence of the electric field $\mathcal{E}(t)$, i.e., by solving the timedependent Schrödinger equation,

$$
i \hbar \frac{\partial}{\partial t} \Psi(t)=[\hat{H}(\mathcal{E}(t))] \Psi(t), \quad \Psi(0)=\Phi_{i} .
$$

The "target" state can be defined by

$$
\Phi_{f}=\frac{\hat{\mathrm{O}} \Psi(T)}{\|\hat{\mathrm{O}} \Psi(T)\|},
$$

where the target operator Ô is specified in Sec. II C. Following the original TBQCP method, ${ }^{15}$ we introduce a wave function $\chi(t)$ associated with a reference control field $\mathcal{E}^{(0)}(t)$ and final state $\Phi_{f}$, which satisfies the time-dependent Schrödinger equation,

$$
i \hbar \frac{\partial}{\partial t} \chi(t)=\left[\hat{H}\left(\mathcal{E}^{(0)}(t)\right)\right] \chi(t), \quad \chi(T)=\Phi_{f} .
$$

As suggested in Ref. 16, we can then choose the following expression:

$$
\mathcal{E}(t)=\mathcal{E}^{(0)}(t)+\eta s(t) \mathcal{E}_{\alpha}(t)
$$

for the control field $\mathcal{E}(t)$, where

$$
\mathcal{E}_{\alpha}(t)=-\frac{2}{\hbar} \operatorname{Im}\left\{\frac{\langle\Psi(t) \mid \chi(t)\rangle}{|\langle\Psi(t) \mid \chi(t)\rangle|^{\alpha}} \times\langle\chi(t)|\mu| \Psi(t)\rangle\right\},
$$

$0 \leq \alpha \leq 1$. The parameter $\eta>0$ designates the size of the change from $\mathcal{E}^{(0)}(t)$ to $\mathcal{E}(t)$ (note that $\eta$ has a unit such that $\eta \mathcal{E}_{\alpha}(t)$ has the unit of an electric field). A large value $\eta$ results in large modifications in the field, while a small value of $\eta$ represents a conservative search strategy with only small modifications in the field at each iteration. The shape function $s(t)$ was chosen as $s(t)=\sin ^{2}(\pi t / T)$ to enforce a smooth switch on and off of the field. The choice of control fields in Eq. (7) implies an update rule rather than a replacement rule in the control equation for the field. Physically, it corresponds to minimizing the change in pulse energy at each iteration. ${ }^{21}$ The system of coupled equations, Eqs. 4, 6, and 7, is solved using an iterative method starting with an arbitrary reference field $\mathcal{E}^{(0)}(t) .{ }^{8}$ Due to the initial conditions, Eqs. (4) and (6) correspond to a forward and a backward propagation in time, respectively. The design of the optimal pulse requires typically an intensive iteration process to modify the initial optical pulse to achieve the desired objective. Further details of this fast-kick-off TBQCP method can be found in Ref. 16.

OCT is a powerful method to predict pulse structures such that the system is steered towards a desired state or a reaction channel, however, if we do not introduce any constraints on the optimized pulses, the fields obtained with standard OCT using iterative methods are often complex both in temporal shape and spectral composition, making it difficult to produce such a field experimentally or to understand the mechanism induced by the field. Thus, the resultant control field $\mathcal{E}(t)$ can be further filtered to remove unwanted low- and high-frequency components by invoking the relation

$$
\mathcal{E}(t)=\mathcal{F}^{-1}\{\mathcal{F}[\mathcal{E}(\cdot)] h(\omega)\}
$$

at the end of each iteration, where $\mathcal{F}$ and $\mathcal{F}^{-1}$ are, respectively, Fourier and inverse Fourier transforms, $\mathcal{E}(\cdot)$ is the electric field before Fourier transformation to frequency space, and $h(\omega)$ is any proper bandpass filter function.

In our calculations, we assume that the $\mathrm{CH}_{2} \mathrm{BrCl}$ molecule is initially prepared in the vibrational ground state of the electronic ground state, and the initial wave function $\Phi_{i}$ is calculated with the Fourier Grid Hamiltonian method. ${ }^{35}$ The wave packet propagation is carried out in a grid of 512 $\times 512$ points with a grid spacing of $0.01 \AA$. The action of the kinetic energy operator is evaluated by the fast Fouriertransform technique, and the time propagation is done by the split-operator method ${ }^{36,37}$ with a time step of 0.02 fs. All calculations in the following are performed within the atomic system of units (a.u.) and unless otherwise specified, numbers are reported within this unit system (that is, energies are in the unit of hartree, dipole moments are in the unit of bohr radius times elementary charge, electric fields are in the unit of hartree/[bohr radius times elementary charge], and time/frequency is measured in the unit of $2.41888 \times 10^{-17} \mathrm{~s}$ ). 
The target time $T$ is set to $60 \mathrm{fs}$, such that the wave packet always stayed within the grid region. The initial pulse is chosen as $\mathcal{E}(t)=E_{0} \sin ^{2}\left(\pi t / t_{p}\right) \cos \omega t$ with duration of the laser pulse $t_{p}=20 \mathrm{fs}$, central frequency $\omega=0.2574$ a.u. (wavelength $177 \mathrm{~nm}$ ), and the amplitude $E_{0}=7.549 \times 10^{-4}$ a.u. corresponding to a peak intensity of $2.0 \times 10^{10} \mathrm{~W} / \mathrm{cm}^{2}$. The filter function $h(\omega)$ was chosen as the Butterworth bandpass filter ${ }^{15}$ of order 120 with frequency range from 0.05 to 0.37 a.u. The time-dependent Schrödinger equation within the control equations is solved numerically to obtain the wave function $\Psi\left(r_{1}, r_{2}, t\right)$, which is a three-component vector, $\Psi\left(r_{1}\right.$, $\left.r_{2}, t\right)=\left[\Psi_{0}\left(r_{1}, r_{2}, t\right), \Psi_{1}\left(r_{1}, r_{2}, t\right), \Psi_{2}\left(r_{1}, r_{2}, t\right)\right]^{T}$, each component representing the part of the wave function on the related diabatic electronic state.

\section{Target operator}

For wavelengths below $180 \mathrm{~nm}$, the majority of the molecules will be excited to the second diabatic excited state (adiabatic $\mathrm{c}^{1} A^{\prime}$ state) upon vertical transition. Since there exists a strong coupling, see Fig. 2(a), between the excited states in the FC region which lies entirely on the $r_{1}>r_{2}$ side, the population on $V_{2}$ will partially transfer to $V_{1}$ (adiabatic $\mathrm{b}^{1} A^{\prime}$ state) by nonadiabatic coupling. Once the $\mathrm{C}-\mathrm{Br}(\mathrm{C}-\mathrm{Cl})$ bond length in the diabatic state $V_{1}\left(V_{2}\right)$ reaches a distance of about $4 \AA$, it will irreversibly dissociate along the $\mathrm{C}-\mathrm{Br}(\mathrm{C}-\mathrm{Cl})$ coordinate. The $\mathrm{Br} / \mathrm{Cl}$ branching ratio is mainly determined by the nonadiabatic transition. ${ }^{31}$ It should be mentioned that the two diabatic excited states in Fig. 2(b) are almost degenerate in the $\mathrm{FC}$ region, and the $V_{2}\left(r_{2}>r_{1}\right)$ is lower than $V_{1}\left(r_{2}>r_{1}\right)$ in the $\mathrm{CH}_{2} \mathrm{Br}+\mathrm{Cl}$ channel.

Based on these characteristics, the target operator Ô adapted to the present study should be a projection operator that will effectively suppress the wave packet in the coupling region and become a constant of 1 in the asymptotic region corresponding to $\mathrm{CH}_{2} \mathrm{Br}+\mathrm{Cl}$. This is achieved by the following definitions:

$$
\hat{\mathrm{O}}=\left[\begin{array}{ccc}
\beta_{0} \hat{\mathrm{O}}_{0} & 0 & 0 \\
0 & \beta_{1} \hat{\mathrm{O}}_{1} & 0 \\
0 & 0 & \beta_{2} \hat{\mathrm{O}}_{2}
\end{array}\right],
$$

where the operator $\hat{\mathrm{O}}_{\mathrm{j}}(j=0,1,2)$ is a projection operator for the different electronic states. The weights $\beta_{j}$ are chosen to balance the different objectives. Since, ideally, we want to obtain both a high selectivity and a high yield, the target operator is 1 in the target region where all population ends up in the $\mathrm{CH}_{2} \mathrm{Br}+\mathrm{Cl}$ channel of the second excited state. Thus, $\beta_{0}$ $=\beta_{1}=0, \beta_{2}=1$, and $\hat{\mathrm{O}}_{2}$ is chosen as ${ }^{38}$

$$
\hat{\mathrm{O}}_{2}\left(r_{1}, r_{2}\right)=f\left(r_{2}, r_{2}^{a}, r_{2}^{b}\right)\left(1-f\left(r_{1}, r_{1}^{a}, r_{1}^{b}\right)\right)
$$

with

$$
f(x, y, z)= \begin{cases}0, & x<y \\ \sin ^{2} \frac{\pi(x-y)}{2(z-y)}, & y \leq x \leq z \\ 1, & x>z,\end{cases}
$$

Figure 2(c) shows the target operator $\hat{\mathrm{O}}_{2}$ with $r_{1}^{a}=3.5, r_{1}^{b}$ $=4.0, r_{2}^{a}=2.5$, and $r_{2}^{b}=3.0 \AA$.
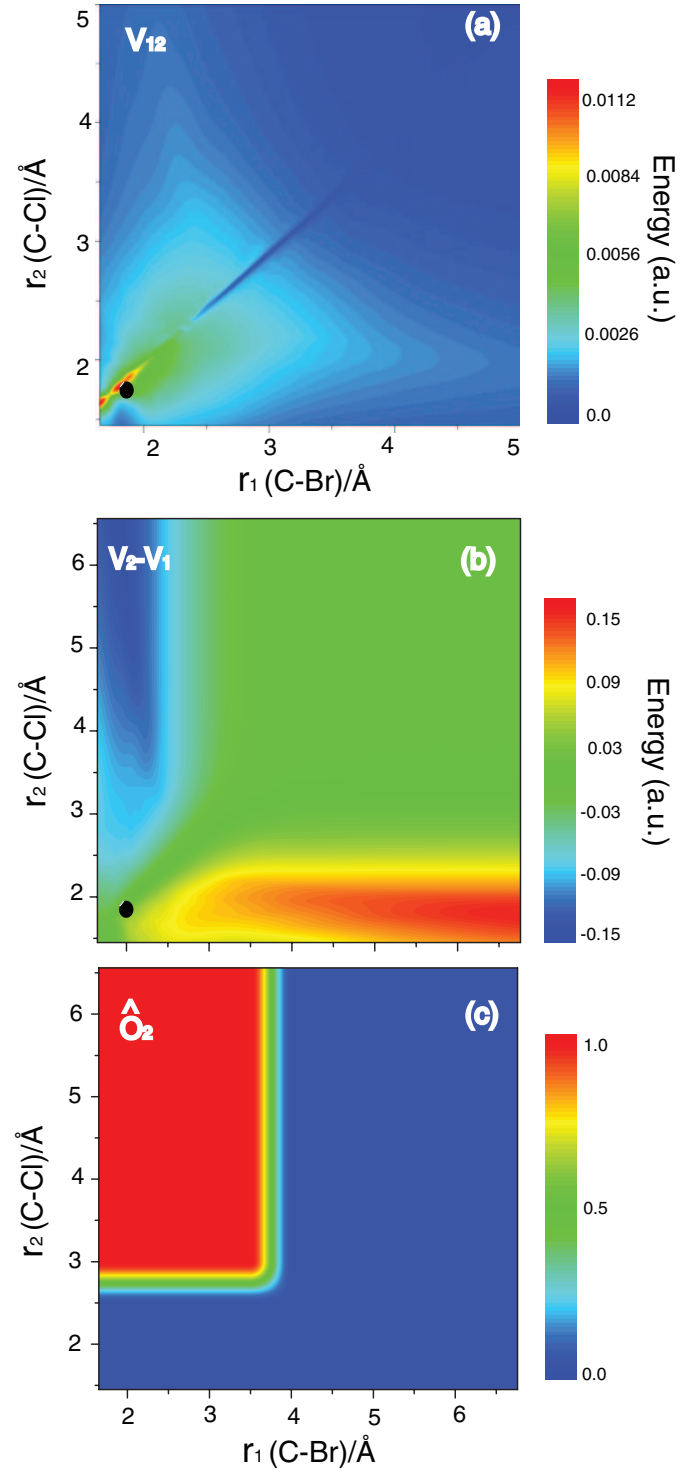

FIG. 2. Potential coupling $V_{12}$, the difference potential $V_{2}-V_{1}$, and the target operator $\hat{\mathrm{O}}_{2}$. The black dots mark the position of the vibrational ground state, i.e., the Franck-Condon region.

\section{RESULTS AND DISCUSSION}

The $a b$ initio values for the transition dipole moments show a quite complex coordinate dependence. Furthermore, the signs of $\mu_{01}$ and $\mu_{02}$ can differ (see Ref. 32, Figs. 3(g)3(h)). As discussed in Sec. III C, this turns out to give an extremely slow convergence of the OCT algorithm. Therefore, we shall first consider various simple approximations to the transition dipole moment surfaces.

\section{A. OCT optimization with constant transition dipole moments, $\mu_{01}=\mu_{02}=1$ a.u.}

Figure 3(a) shows the final populations in the $V_{1}$ and $V_{2}$ diabatic states and the population in the target region, as a function of iteration for constant transition dipole moments of $\mu_{01}=\mu_{02}=1.0$ a.u. This assumption has been introduced in many previous applications of OCT (see, e.g., Refs. 2 and 3). The control parameters $\eta$ and $\alpha$ are set to 0.5 and 1.0, 

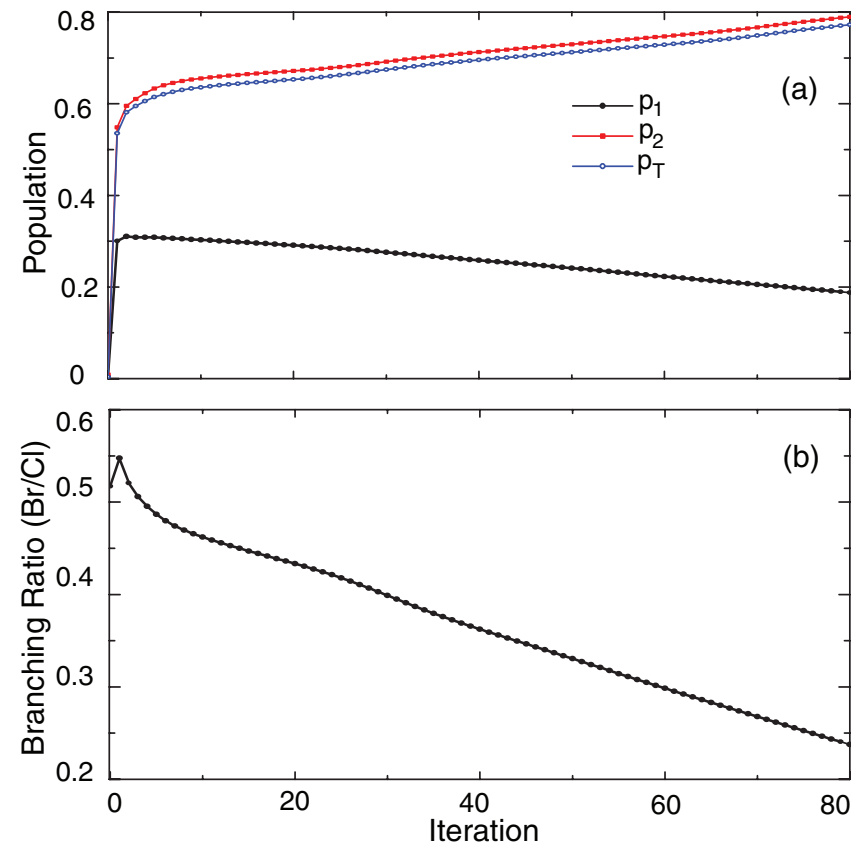

FIG. 3. (a) The final populations on the first $\left(P_{1}\right)$ and second $\left(P_{2}\right)$ excited states and in the target region $\left(P_{T}\right),(b)$ the $\mathrm{Br} / \mathrm{Cl}$ branching ratio as a function of iteration. The transition dipole moments are assumed to be constant with a value of 1 a.u.

respectively, which gives a fast convergence. It should be noted that the pulse energy is changing (increasing) as a function of iteration. It can clearly be seen that the final population in the target region is monotonically increasing. However, the final population on $V_{1}$ increases during the first few iterations, and then decreases as a function of iteration. After about 80 iterations, $\sim 80 \%$ of the molecules are transferred to $V_{2}$ and almost all of them within the target region. The $\mathrm{Br} / \mathrm{Cl}$ branching ratio given by the ratio between the populations in the first and second excited states, i.e., $R=P_{1}(T) / P_{2}(T)$, is clearly lower than 0.4 as shown in Fig. 3(b).

We now analyze the optimized laser pulse and the dynamics after 65 iterations. Figures 4(a) and 4(b) show the time-dependent electric field and the Husimi transform power spectrum of the optimized pulse as a function of time and frequency. There are no frequency components below 0.20 a.u. The time-dependent populations in each electronic state are displayed in Fig. 4(c). It is clear that the optimized laser pulse consists of, at least, four sub-pulses. The first two pulses are positively chirped whereas the last two pulses are negatively chirped. It is noted that the population transfer of the last pulse, centered at around $31 \mathrm{fs}$, seems not to play any significant role (removing this part of the field after $\sim 28$ fs reduces the population transfer to $V_{2}$ by just a few percent). The first two pulses with central frequencies around 0.26 a.u. and 0.31 a.u. transfer the majority of the population to $V_{2}$. The third intense pulse with central frequency around 0.27 a.u. induces a series of quick pump-dump processes between $V_{2}$ and $V_{0}$. The population remaining on the ground state is almost completely pumped to $V_{2}$ with the third negatively chirped pulse. At the final time, more than $99 \%$ of the population has been transferred to the two excited states, in which about $76 \%$ is in $V_{2}$. It indicates that the $\mathrm{Br} / \mathrm{Cl}$ branching ratio cal-
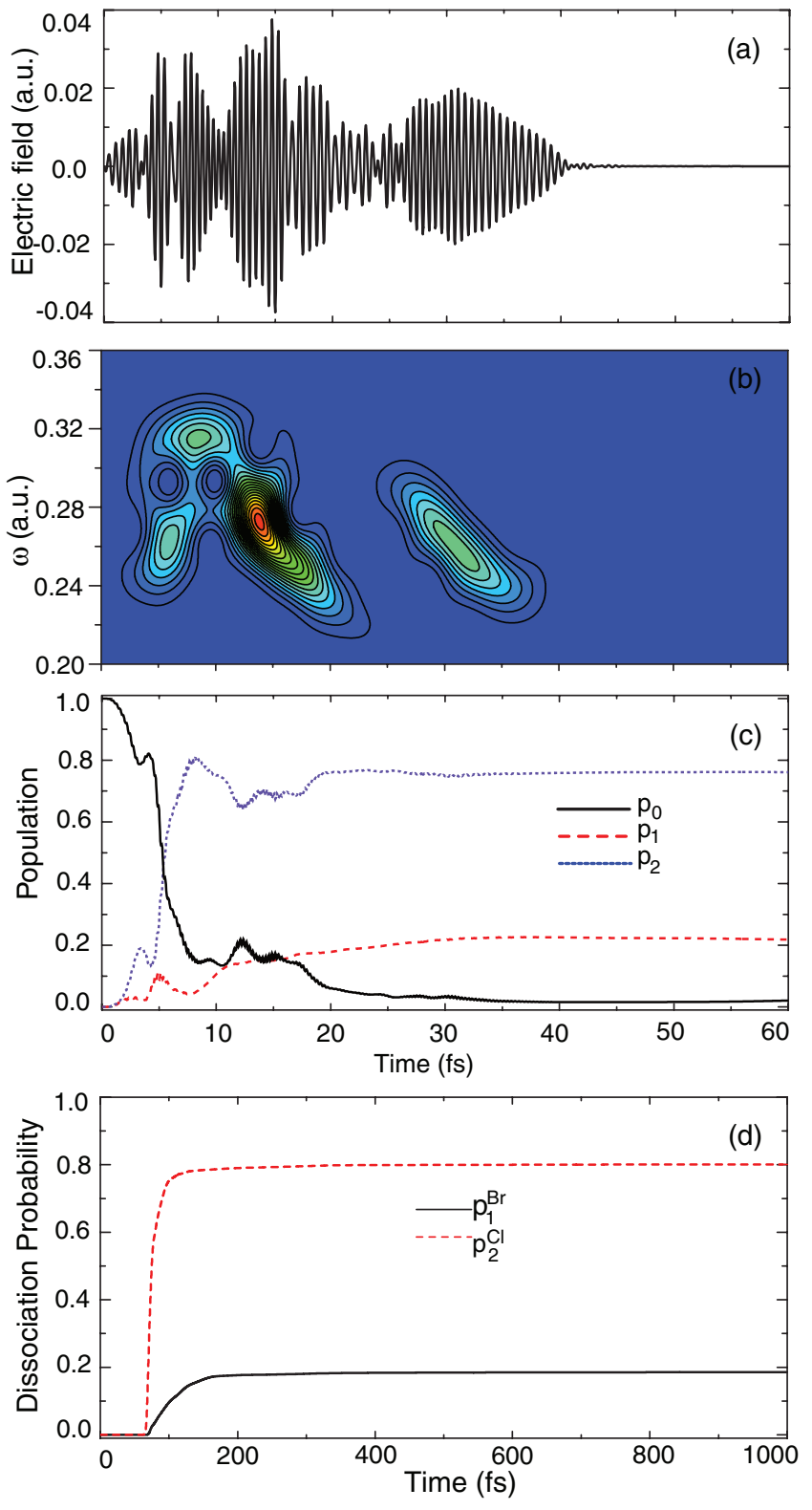

FIG. 4. (a) The time-dependent electric field and (b) the Husimi transform power spectrum as a function of time and frequency for the optimized laser pulse obtained after 65 iterations. (c) Time-dependent populations in $V_{0}\left(P_{0}\right)$, $V_{1}\left(P_{1}\right)$, and $V_{2}\left(P_{2}\right)$. (d) Time-integrated total flux in the channels $\mathrm{CH}_{2} \mathrm{Cl}+$ $\mathrm{Br}$ for the first excited state and $\mathrm{CH}_{2} \mathrm{Br}+\mathrm{Cl}$ for the second excited state. The transition dipole moments are assumed to be constant with a value of 1 a.u.

culated as the population ratio $P_{1} / P_{2}$ has been decreased to about 0.28 . The peak intensity corresponding to the maximum amplitude of the electric field is close to $50 \mathrm{TW} / \mathrm{cm}^{2}$.

To further examine the dissociation, the dissociation probability can be calculated by the time-integrated total flux in the competing channels, $\mathrm{CH}_{2} \mathrm{Cl}+\mathrm{Br}$ and $\mathrm{CH}_{2} \mathrm{Br}+\mathrm{Cl}$, according to the following equations: ${ }^{39}$

$$
\begin{aligned}
P_{k}^{\mathrm{Br}}(t) & =\int_{0}^{t}\left(\int_{0}^{\infty} \Psi_{k}^{*}\left(r_{1}, r_{2}, \tau\right) \hat{j}_{1} \Psi_{k}\left(r_{1}, r_{2}, \tau\right) d r_{2}\right. \\
& \left.+\frac{m_{2} \cos \theta}{m_{3}} \int_{r_{1}^{d}}^{\infty} \Psi_{k}^{*}\left(r_{1}, r_{2}, \tau\right) \hat{j}_{2} \Psi_{k}\left(r_{1}, r_{2}, \tau\right) d r_{1}\right) d \tau
\end{aligned}
$$


and

$$
\begin{aligned}
P_{k}^{\mathrm{Cl}}(t) & =\int_{0}^{t}\left(\int_{0}^{\infty} \Psi_{k}^{*}\left(r_{1}, r_{2}, \tau\right) \hat{j}_{2} \Psi_{k}\left(r_{1}, r_{2}, \tau\right) d r_{1}\right. \\
& \left.+\frac{m_{1} \cos \theta}{m_{3}} \int_{r_{2}^{d}}^{\infty} \Psi_{k}^{*}\left(r_{1}, r_{2}, \tau\right) \hat{j}_{1} \Psi_{k}\left(r_{1}, r_{2}, \tau\right) d r_{2}\right) d \tau
\end{aligned}
$$

where $\hat{j}_{i}$ is the flux operator in the $i$ th channel, defined as

$$
\hat{j}_{i}=\frac{1}{2 m_{i}}\left[\hat{p}_{i} \delta\left(r_{i}-r_{i}^{d}\right)+\delta\left(r_{i}-r_{i}^{d}\right) \hat{p}_{i}\right]
$$

where $\hat{p}_{i}$ is the momentum operator and $r_{i}^{d}$ is an asymptotic point outside of the diabatic coupling region of the $i$ th channel (here $r_{1}^{d}=6.23 \AA$ and $r_{2}^{d}=6.05 \AA$ ). Here, an absorbing ramp potential is used at the asymptotic cuts to avoid unphysical reflection from the edges. ${ }^{40} \mathrm{We}$ plot the time-integrated total flux in the channels, $\mathrm{CH}_{2} \mathrm{Cl}+\mathrm{Br}$ for the first excited state and $\mathrm{CH}_{2} \mathrm{Br}+\mathrm{Cl}$ for the second excited state, as shown in Fig. 4(d). As can be seen from Figs. 4(c) and 4(d), the dissociation probability $\left(P_{2}^{\mathrm{Cl}}=0.8\right)$ at the final time is a little bit higher than the final population $\left(P_{2}(T)=0.76\right)$ in $V_{2}$. This is because the wave packet on the higher excited state $V_{1}\left(r_{2}>r_{1}\right)$ is transferred partially to the lower excited state $V_{2}\left(r_{2}>r_{1}\right)$ by electronic coupling when it turns back to the $r_{1}>r_{2}$ side of the first diabatic potential. Thus, the branching ratio is 0.22 , well below the value which can be obtained in the weak-field limit (using the Hamiltonian of Eq. (2) with any form of UV-pulse shaping).

A key point in the mechanism is the creation of a nonstationary vibrational state in the electronic ground state. Figure 5 shows the wave function (absolute square) in the ground and second excited states $V_{0}$ and $V_{2}$ at some given times. The initial wave function in the ground state lies entirely on the $r_{1}>r_{2}$ side, as shown in Fig. 5(a). Within our reduced pseudo-triatomic model, the vibrational period of the lowest $\mathrm{C}-\mathrm{Cl}$ stretching mode is about $44 \mathrm{fs}$. We observe that the population transfer is completed within about half of this time. After about half of the vibrational period, the majority of the population in $V_{2}$ has gone into the $r_{2}>r_{1}$ region, and a nonstationary vibrational state is created in the electronic ground state, as shown in Figs. 5(c) and 5(e). When the nonstationary vibrational state is stretched to an appropriate position and with a dominant momentum in the $\mathrm{C}-\mathrm{Cl}$ direction, the wave packet in the ground state is quickly pumped to $V_{2}$, and turned into the target region for dissociation, which suppresses the effect of nonadiabatic transition to $V_{1}$, as shown in Figs. 5(d) and 5(f).

This scenario is similar to the two-pulse control scheme suggested some time ago, ${ }^{41,42}$ and demonstrated recently for $\mathrm{CH}_{2} \mathrm{BrCl}^{43}$ There, an intense few-cycle infrared (IR) laser pulse was used to directly create a nonstationary vibrational state in the electronic ground state, and the following UV laser pulse excited the wave packet to the target region of the $V_{2}$ potential.
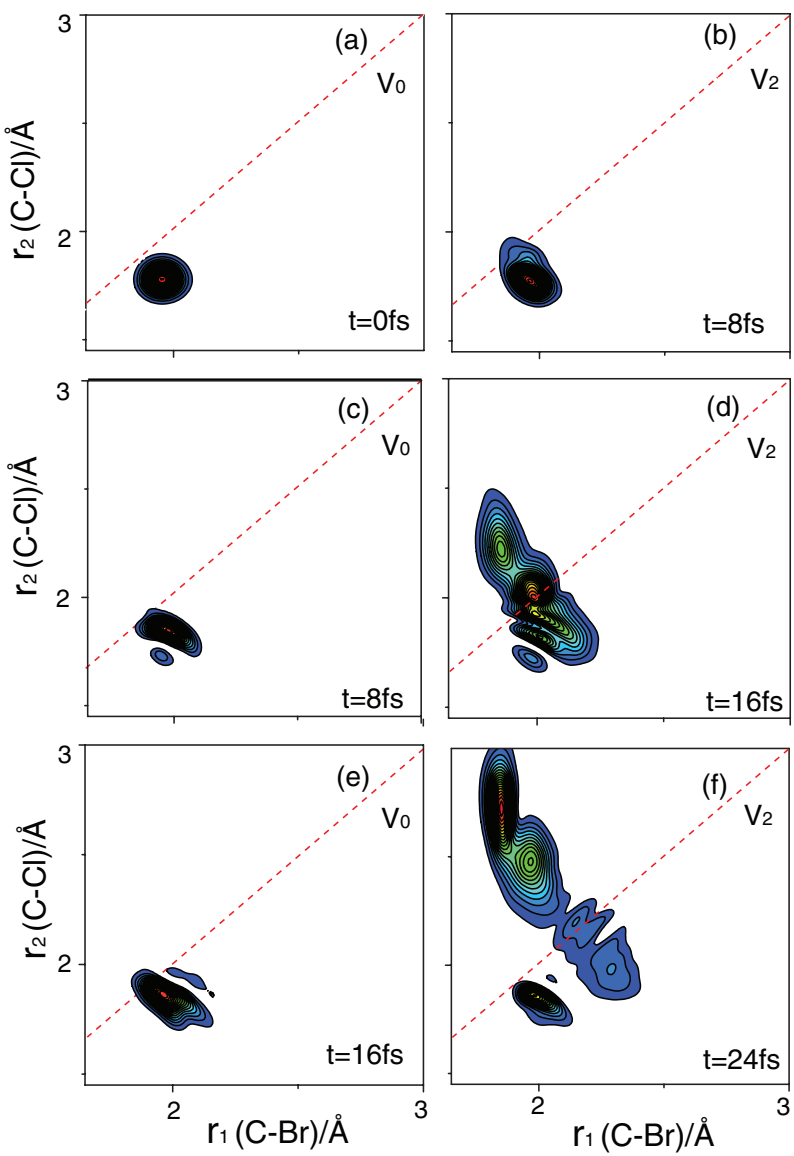

FIG. 5. Plots of the vibrational probability density in the ground and the second excited state during the action of the pulse in Fig. 4(a). The dashed lines indicate that $r_{1}=r_{2}$. The transition dipole moments are assumed to be constant with a value of 1 a.u.

\section{B. OCT optimization with the constant transition dipole moments, $\left|\mu_{01}\right|=0.185$ a.u. and $\left|\mu_{02}\right|=0.275$ a.u. at vertical excitation}

We consider now a more realistic approximation to the transition dipole moment surfaces. We assume again constant values (i.e., the Condon approximation) but use the calculated values at vertical excitation, ${ }^{32}\left|\mu_{01}\right|=0.185$ a.u. and $\left|\mu_{02}\right|$ $=0.275$ a.u. We note that with the introduction of different signs for $\mu_{01}$ and $\mu_{02}$, as obtained from the electronic structure calculations, the convergence of the OCT algorithm is so slow that the calculation is not feasible.

Figure 6(a) shows the final populations in the $V_{1}$ and $V_{2}$ diabatic states, and the population in the target region as a function of iteration with the control parameters $\eta$ and $\alpha$ set to 4 and 1.0, respectively. The calculated results are very similar to that in Fig. 3, with the branching ratio decreased to close to 0.3 after about 80 iterations. As an example, we consider in the following the dynamics after 83 iterations, where the branching ratio has been decreased to 0.3 , evaluated as the ratio between the populations in the first and second excited state. Figure 7 shows the optimized electric field and the associated time evolution in the populations. Positive chirp is observed in the first part of the pulse, followed by a negatively chirped part. Again, the last part of the pulse centered around 

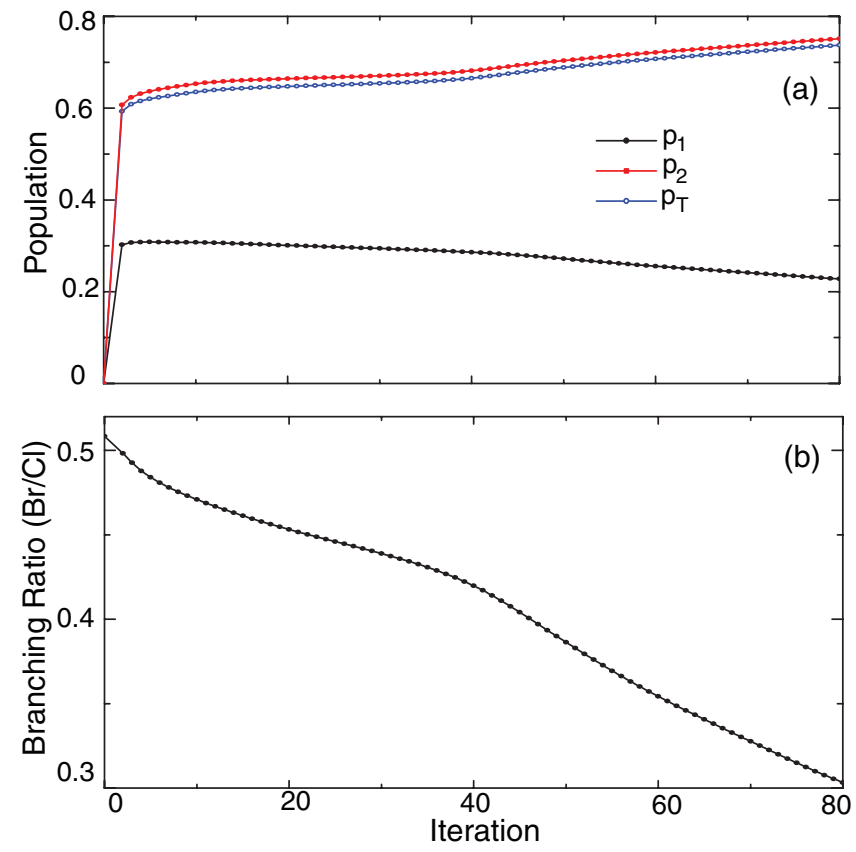

FIG. 6. (a) The final populations on the first and second excited states and in the target region, (b) the $\mathrm{Br} / \mathrm{Cl}$ branching ratio as a function of iteration with the constant transition dipole moments $\left|\mu_{01}\right|=0.185$ a.u. and $\left|\mu_{02}\right|=0.275$ a.u. corresponding to the $a b$ initio values at vertical excitation.

$31 \mathrm{fs}$ seems not to play any significant role in the population transfer.

As in Fig. 4(d), the branching ratio was also examined by calculating the time-integrated total flux, which gives a branching ratio of 0.25 with about $20 \%$ population in the first diabatic excited state and $80 \%$ population in the second diabatic state. However, laser intensities are extremely high with a peak intensity corresponding to the maximum amplitude of the electric field close to $10^{3} \mathrm{TW} / \mathrm{cm}^{2}$ and an average intensity around $200 \mathrm{TW} / \mathrm{cm}^{2}$ in the time window from 10 to $20 \mathrm{fs}$. These high values are a consequence of the small transition dipole moments. Such high laser intensities are a problem, since they will lead to extensive ionization. For example, photoionization with $800 \mathrm{~nm}$ pulses leading to singly charged parent ions and subsequent fragmentation has been observed for organic hydrocarbons ${ }^{44,45}$ and for $\mathrm{CH}_{2} \mathrm{BrI}$ and $\mathrm{CH}_{2} \mathrm{Br}_{2}{ }^{46,47}$ at laser peak intensities of about $100 \mathrm{TW} / \mathrm{cm}^{2}$.

\section{OCT optimization with real transition dipole moment surfaces}

With the present computational resources the application of OCT algorithms is limited to low-dimensional systems. Even with the new "fast-kick-off" TBQCP method, ${ }^{16}$ we find that the convergence of the method for $\mathrm{CH}_{2} \mathrm{BrCl}$ is so slow that the calculation is not feasible when the real coordinate dependent transition dipole moment surfaces are used. As mentioned above, the slow convergence was apparent already with the introduction of different signs for $\mu_{01}$ and $\mu_{02}$, as obtained from the electronic structure calculations.

It should be noted that it is the frequency constraint in Eq. (9) which reduces the convergence rate to a level where
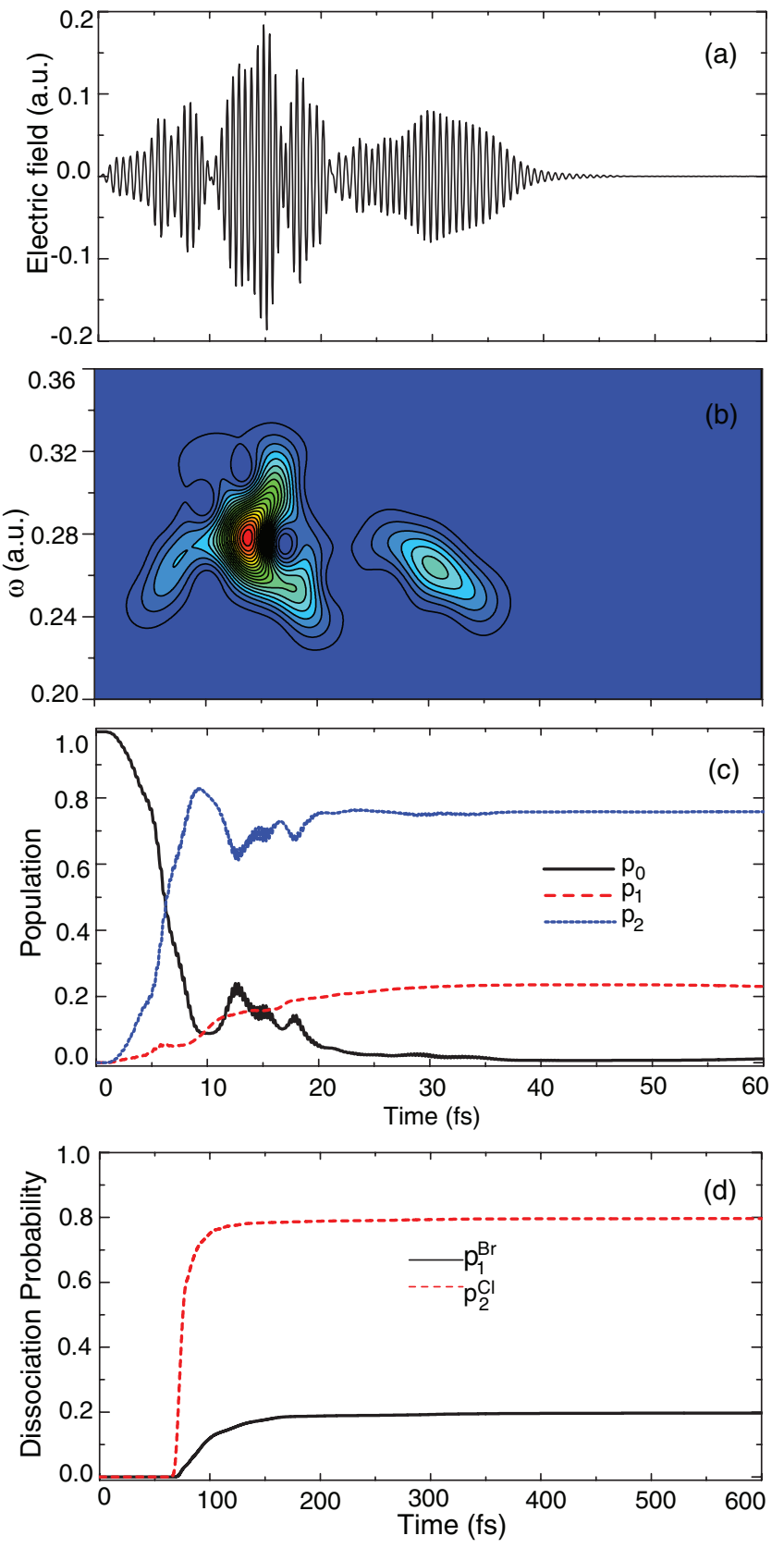

FIG. 7. (a) The time-dependent electric field and (b) the Husimi transform power spectrum as a function of time and frequency for the optimized laser pulse obtained after 83 iterations. (c) Time-dependent populations in $V_{0}$ $\left(P_{0}\right), V_{1}\left(P_{1}\right)$, and $V_{2}\left(P_{2}\right)$. (d) Time-integrated total flux in the channels $\mathrm{CH}_{2} \mathrm{Cl}+\mathrm{Br}$ for the first excited state and $\mathrm{CH}_{2} \mathrm{Br}+\mathrm{Cl}$ for the second excited state. Constant transition dipole moments $\left|\mu_{01}\right|=0.185$ a.u. and $\left|\mu_{02}\right|$ $=0.275$ a.u. are assumed corresponding to the $a b$ initio values at vertical excitation.

the calculation is not feasible. When the frequency filtering is removed, a branching ratio close to 0.25 is obtained after 67 iterations (similar to the situation with constant transition dipole moments). Figure 8 shows the corresponding optimized electric field. The first part of the pulse is similar to that in Fig. 7, but the subsequent high amplitude part which includes several pump-dump steps is now replaced by a very low-frequency (dc) component which can (Stark) shift the potential energy surfaces. Such dc and low-frequency components in optimized fields without bandwidth restrictions have 


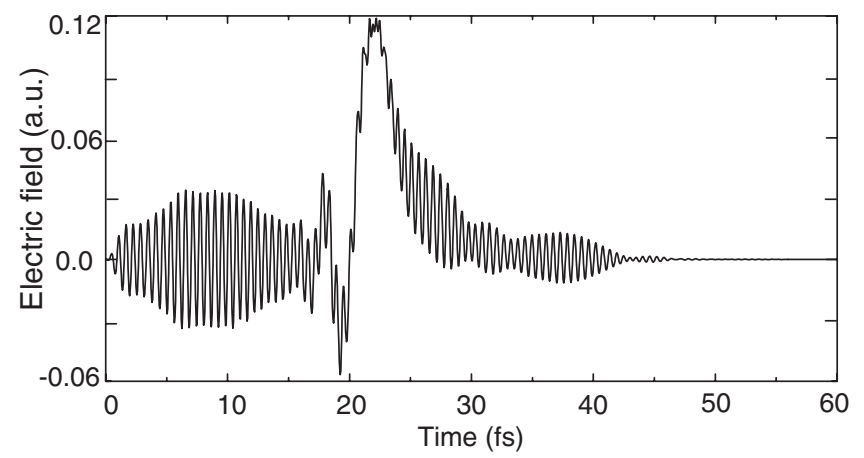

FIG. 8. The optimized time-dependent electric field using the real transition dipole moment surfaces. Note that the frequency filter has been removed.

been observed before, e.g., in curve-crossing systems. ${ }^{48}$ However, the maximum amplitude is still very high. (We note that the electric field in Fig. 8 does not strictly fulfill the condition of a time integral which is equal to zero. This might be fixed by introducing a new constraint on the electric field, at the risk of making the calculation unfeasible due to slow convergence.)

The complexity of the system and the small values of the transition dipole moments imply that very high intensities are unavoidable. Thus, even if a converged result could be obtained using the real transition dipole moment surfaces, including appropriate frequency filtering, the result will most likely be a laser field with an intensity where ionization plays an essential role.

\section{CONCLUSIONS}

We have analyzed the laser-induced control of the $\mathrm{Br} / \mathrm{Cl}$ branching ratio, $\mathrm{Br}+\mathrm{CH}_{2} \mathrm{Cl} \leftarrow \mathrm{CH}_{2} \mathrm{BrCl} \rightarrow \mathrm{CH}_{2} \mathrm{Br}+\mathrm{Cl}$, within the $\tilde{\mathrm{B}}$ absorption band of $\mathrm{CH}_{2} \mathrm{BrCl}$. The $\mathrm{CH}_{2} \mathrm{BrCl}$ molecule was initially assumed to be in its vibrational ground state as in most current experiments with shaped laser pulses. The description of the nonadiabatic nuclear quantum dynamics was based on high level ab initio potential energy surfaces and realistic transition dipole moments. Optimized laser pulses in the UV region were obtained via the optimal control theory.

Due to the complexity of the system, which includes three electronic states, nonadiabatic couplings, and complicated transition dipole moments surfaces, we found that the convergence is so slow that a desired $\mathrm{Br} / \mathrm{Cl}$ branching ratio (less than 0.4) could not be obtained. However, a laser pulse which leads to breakage of the $\mathrm{C}-\mathrm{Cl}$ bond with a selectivity beyond what is obtainable with a narrow-band (or any shaped) UV laser in the weak-field limit could be obtained quite readily, if constant transition dipole moment surfaces were employed. The duration of the optimized pulse is about $50 \mathrm{fs}$ and it consists essentially of two subpulses. The pulses centered at about $170 \mathrm{~nm}$ lead essentially to a mechanism reminiscent of a pump-dump-pump mechanism between the electronic ground state and the second excited diabatic state. During this process, a nonstationary vibrational state is created in the electronic ground state of $\mathrm{CH}_{2} \mathrm{BrCl}$.
The model study involving large values (1 a.u.) of the transition dipole moments results in an electric field of high but not exceedingly large intensity. However, the model study based on more realistic constant transition dipole moment surfaces with values equal to the $a b$ initio values at vertical excitation or the use of the real transition dipole moment surfaces (without frequency filter) strongly suggests that control using the present approach is not possible without the application of laser intensities which will lead to substantial ionization.

We considered the objective of a high yield of $\mathrm{CH}_{2} \mathrm{Br}$ $+\mathrm{Cl}$ as well as a the high $\mathrm{Br} / \mathrm{Cl}$ selectivity. The intensities might perhaps be lowered somewhat if the requirement of high yield is reduced. However, in order to beat the selectivity obtained in the weak-field limit via the Tannor-Rice type mechanism investigated in the present work - very high intensities are required. At these intensities ionization will play a non-negligible role. Thus, the present work strongly suggests that a Tannor-Rice type laser control mechanism for selective bond breakage in $\mathrm{CH}_{2} \mathrm{BrCl}$ cannot take place without accompanying photoionization.

An alternative approach to selective bond breakage in $\mathrm{CH}_{2} \mathrm{BrCl}$ involves a direct vibrational excitation using an IR pulse followed by a UV pulse, which excites the molecule into the second excited electronic state ${ }^{41-43}$ (see also Ref. 49). In this approach a high intensity IR pulse is required but the intensity is substantially lower (i.e., of the order of $5 \mathrm{TW} / \mathrm{cm}^{2}$ ) than in the mechanism studied in the present work. ${ }^{43}$ Furthermore, no loss of selectivity is experienced due to the first pump-dump step via the second excited electronic state. Accordingly, a very high selectivity can be obtained, such as $\mathrm{Br} / \mathrm{Cl} \sim 0.05 .{ }^{43}$

\section{ACKNOWLEDGMENTS}

This work was supported by The Technical University of Denmark through a scholarship under the H.C. Ørsted Postdoc Programme, the European COST action CM0702, and by the Hungarian-German project 436 UNG 113/188/0-1 financed by the Deutsche Forschungsgemeinschaft (DFG).

${ }^{1}$ D. J. Tannor and S. A. Rice, J. Chem. Phys. 83, 5013 (1986).

${ }^{2}$ D. J. Tannor, R. Kosloff, and S. A. Rice, J. Chem. Phys. 85, 5805 (1986).

${ }^{3}$ R. Kosloff, S. A. Rice, P. Gaspard, S. Tersigni, and D. J. Tannor, Chem. Phys. 139, 201 (1989).

${ }^{4}$ P. Brumer and M. Shapiro, Chem. Phys. Lett. 126, 541 (1986).

${ }^{5}$ W. Jakubetz, B. Just, and J. Manz, J. Chem. Phys. 94, 2294 (1990).

${ }^{6}$ R. Judson and H. Rabitz, Phys. Rev. Lett. 68, 1500 (1992).

${ }^{7}$ C. Brif, R. Chakrabarti, and H. Rabitz, New J. Phys. 12, 075008 (2010).

${ }^{8}$ J. Werschnik and E. K. U. Gross, J. Phys. B 40, R175 (2007).

${ }^{9}$ R. Eitan, M. Mundt, and D. J. Tannor, Phys. Rev. A 83, 053426 (2011).

${ }^{10}$ V. F. Krotov, Autom. Remote Control (Engl. Transl.) 70, 357 (2009).

${ }^{11}$ S. E. Sklarz and D. J. Tannor, Phys. Rev. A 66, 053619 (2002).

${ }^{12}$ W. Zhu and H. Rabitz, J. Chem. Phys. 108, 1953 (1998).

${ }^{13}$ W. Zhu and H. Rabitz, J. Chem. Phys. 109, 385 (1998).

${ }^{14}$ T.-S. Ho, H. Rabitz, and S.-I. Chu, Comput. Phys. Commun. 182, 14 (2011).

${ }^{15}$ T.-S. Ho and H. Rabitz, Phys. Rev. E 82, 026703 (2010).

${ }^{16}$ S.-L. Liao, T.-S. Ho, S.-I Chu, and H. Rabitz, Phys. Rev. A 84, 031401 (2011).

${ }^{17}$ A. K. Tiwari, K. B. Møller, and N. E. Henriksen, Phys. Rev. A 78, 065402 (2008)

${ }^{18}$ C. M. Tesch and R. de Vivie-Riedle, Phys. Rev. Lett. 89, 157901 (2002).

${ }^{19}$ M. Schröder and A. Brown, New J. Phys. 11, 105031 (2009). 
${ }^{20}$ R. R. Zaari and A. Brown, J. Chem. Phys. 135, 044317 (2011).

${ }^{21}$ C. P. Koch, J. P. Palao, R. Kosloff, and F. Masnou-Seeuws, Phys. Rev. A 70, 013402 (2004).

${ }^{22}$ D. J. Tannor and A. Bartana, J. Phys. Chem. A 103, 10359 (1999).

${ }^{23}$ T. Brixner and G. Gerber, Chem. Phys. Chem. 4, 418 (2003).

${ }^{24}$ P. Zou, W. S. McGivern, and S. W. North, Phys. Chem. Chem. Phys. 2, 3785 (2000).

${ }^{25}$ T. Rozgonyi and L. González, J. Phys. Chem. A 106, 11150 (2002).

${ }^{26}$ D. Irimia and M. H. M. Janssen, J. Chem. Phys. 132, 234302 (2010).

${ }^{27}$ L. J. Butler, E. J. Hintsa, S. F. Shane, and Y. T. Lee, J. Chem. Phys. 86, 2051 (1987).

${ }^{28}$ D. G. Abrashkevich, M. Shapiro, and P. Brumer, J. Chem. Phys. 116, 5584 (2002).

${ }^{29}$ S.-H. Lee, Y.-J. Jung, and K.-H. Jung, Chem. Phys. Lett. 260, 143 (2000).

${ }^{30}$ J.-G. Zhou, K.-C. Lau, E. Hassanein, H.-F. Xu, S.-X. Tian, B. Jones, and C. Y. Ng, J. Chem. Phys. 124, 034309 (2006).

${ }^{31}$ T. Rozgonyi and L. González, J. Phys. Chem. A 110, 10251 (2006).

${ }^{32}$ T. Rozgonyi and L. González, J. Phys. Chem. A 112, 5573 (2008).

${ }^{33}$ G. Katz, K. Yamashita, Y. Zeiri, and R. Kosloff, J. Chem. Phys. 116, 4403 (2002).

${ }^{34}$ T. Seideman and E. Hamilton, Adv. At., Mol., Opt. Phys. 52, 289 (2006).
${ }^{35}$ C. C. Marston and G. G. Balint-Kurti, J. Chem. Phys. 91, 3571 (1989).

${ }^{36}$ R. Kosloff, J. Phys. Chem. 92, 2087 (1988).

${ }^{37}$ Z. Sun, N. Lou, and G. Nyman, J. Phys. Chem. A 108, 9226 (2004).

${ }^{38}$ R. de Vivie-Riedle, K. Sundermann, and M. Motzkus, Faraday Discuss. 113, 303 (1999).

${ }^{39}$ M. Sarma, S. Adhikari, and K. Mishra, J. Phys. Chem. A 112, 13302 (2008).

${ }^{40}$ S. Sharma, H. Singh, J. N. Harvey, and G. G. Balint-Kurti, J. Chem. Phys. 133, 174103 (2010).

${ }^{41}$ B. Amstrup and N. E. Henriksen, J. Chem. Phys. 97, 8285 (1992).

${ }^{42}$ B. Amstrup and N. E. Henriksen, J. Chem. Phys. 105, 9115 (1996).

${ }^{43}$ T. Rozgonyi and L. González, Chem. Phys. Lett. 459, 39 (2008).

${ }^{44}$ S. M. Hankin, D. M. Villeneuve, P. B. Corkum, and D. M. Rayner, Phys. Rev. Lett. 84, 5082 (2000).

${ }^{45}$ M. Castillejo, S. Couris, E. Koudoumas, and M. Martín, Chem. Phys. Lett. 308, 373 (1999)

${ }^{46}$ Z.-H. Loh and S. R. Leone, J. Chem. Phys. 128, 204302 (2008).

${ }^{47}$ B. J. Pearson, S. R. Nichols, and T. Weinacht, J. Chem. Phys. 127, 131101 (2007).

${ }^{48}$ P. Gross, D. Neuhauser, and H. Rabitz, J. Chem. Phys. 96, 2834 (1992).

${ }^{49}$ M. Shapiro and P. Brumer, J. Chem. Phys. 98, 201 (1993). 